\title{
Efficacy and safety of homoharringtonine plus cytarabine and aclarubicin for patients with myelodysplastic syndrome-RAEB
}

\author{
FENG XIAO, YING LI, WEILAI XU, LIANGSHUN YOU, CHUNMEI YANG, HUI LIU and WENBIN QIAN \\ Department of Hematology, The First Affiliated Hospital, College of Medicine, \\ Zhejiang University, Hangzhou, Zhejiang 310003, P.R. China
}

Received July 30, 2014; Accepted October 15, 2015

DOI: $10.3892 / \mathrm{ol} .2015 .3876$

\begin{abstract}
The aim of the present study was to evaluate the treatment outcome of homoharringtonine, cytarabine (AraC) and aclarubicin combination therapy as induction treatment for myelodysplastic syndromes-refractory anemia with excess blasts (MDS-RAEB). A total of 24 patients with MDS-RAEB who were aged between 18 and 66 years were treated with homoharringtonine, AraC and aclarubicin (HAA regimen). The HAA regimen consisted of homoharringtonine (2 $\mathrm{mg} / \mathrm{m}^{2}$ intramuscularly twice daily, days 1-3), AraC (75 $\mathrm{mg} / \mathrm{m}^{2}$ injected subcutaneously twice daily, days 1-7) and aclarubicin (12 mg/m² , days 1-7). The overall response rate was $79 \%$ with a complete remission rate of $58.3 \%$ and partial remission rate of $20.7 \%$. There was no evidence of early mortality in this group of patients. The median overall survival (OS) was 36.2 months (95\% confidence interval, 24.6-47.4 months), and the estimated three year overall survival rate was $45.8 \%$. In conclusion, HAA combination therapy is a suitable induction regimen for patients with MDS-RAEB, which may improve the outcome for de novo higher-risk MDS patients, particularly of those with favorable and intermediate cytogenetics.
\end{abstract}

\section{Introduction}

The myelodysplastic syndromes (MDS) are a heterogeneous group of clonal hematopoietic stem-cell disorders characterized by abnormal differentiation, morphology, and maturation of hematopoietic cells in bone marrow (1). Patients with higher-risk MDS, which include intermediate-2 and high risk of the International Prognostic Scoring System (IPSS), have a peculiar propensity to evolve into acute myeloid

Correspondence to: Professor Wenbin Qian, Department of Hematology, The First Affiliated Hospital, College of Medicine, Zhejiang University, 79 Qingchun Road, Hangzhou, Zheijiang 310003, P. R. China

E-mail: qianwenb@hotmail.com

Key words: myelodysplastic syndromes, high-intensity chemotherapy, homoharringtonine, aclarubicin, cytarabine leukemia (AML), and often have a median survival time of $<12$ months $(2,3)$. Currently, hypomethylating agents (such as azacitidine and decitabine), lenalidomide, intensive chemotherapy, and allo-hematopoietic stem cell transplantation may be therapeutic options for patients with higher-risk MDS (4). However, high-intensity chemotherapy is generally reserved for higher-risk patients, particularly young patients eligible for intensive therapy that lack a suitable stem cell donor $(2,5)$. Unfortunately, treatment of MDS patients with chemotherapy only results in a few long-term survivors $(2,5,6)$. Therefore, innovative approaches for the treatment of higher-risk MDS should be developed.

Homoharringtonine (HHT), a plant alkaloid isolated from the Chinese evergreen Cephalotaxus harringtonia, was first investigated in China and has been used for the treatment of chronic myeloid leukemia, AML, and MDS (7-12). It was reported that HHT functions as a protein synthesis inhibitor at the initiation and elongation phases of translation, and that it is a potent apoptosis inducer for hematopoietic malignancies such as AML (13-15). In vitro experiments have demonstrated that HHT inhibited a variety of antiapoptotic proteins including Mcl-1, xIAP and Bcl-2, downregulated Akt pathway and sensitized AML cells to TRAIL-induced apoptosis via increase of death receptor 4 (DR4) and DR5 (13,15-20). Our previous pilot study revealed that HHT combined with cytarabine (AraC) and aclarubicin (HAA regimen) resulted in a complete remission (CR) rate of $83 \%$ in the patients with de novo AML and an estimated 3-year overall survival rate of $53 \%$, which is more effective than any other induction remission regimens currently available (11). Therefore, in the present study, the same regimen was used to treat patients with MDS-refractory anemia with excess blasts (RAEB). The current study presents a retrospective analysis of the outcome of the HAA regimen as induction chemotherapy in the patients with MDS-RAEB.

\section{Patients and methods}

Patients. Between January 2005 and June 2012, 24 patients with MDS-RAEB diagnosed according to the World Health Organization (WHO) classification (21) were enrolled into the presentstudy. The study was approved by the Ethics Committee of the First Affiliated Hospital, College of Medicine, Zhejiang University (Hangzhou, China). All the patients 
Table I. Patient characteristics.

\begin{tabular}{ll}
\hline Characteristics & \multicolumn{1}{c}{$\mathrm{n}$ (range) } \\
\hline Patients & 24 \\
Male/female & $9 / 15$ \\
Median age & 41 years $(18-66)$ \\
FAB classification subtype & \\
$\quad$ RAEB I & 9 \\
RAEB II & 15 \\
Median WBC count & $3.15 \times 10^{9} / 1(1.0-13.3)$ \\
Median platelet count & $53.5 \times 10^{9} / 1(7-198)$ \\
Median hemoglobin & $7.4 \mathrm{~g} / \mathrm{dl}(3.5-11.0)$ \\
Median marrow blast $\%$ & $10.75 \%(6-19)$ \\
Cytogenetics $(\%)$ & \\
Intermediate risk & $18(81.8)$ \\
$\quad$ High risk & $4(18.2)$ \\
$\quad$ Not available & 2 \\
\hline
\end{tabular}

FAB classification, French-American-British classification (22); RAEB, refractory anemia with excess blasts; WBC, white blood cells .

Table II. Complete remission analyses.

\begin{tabular}{lccc}
\hline Characteristics & CR/total & CR rate (\%) & P-value \\
\hline Age & & & 0.665 \\
$<50$ & $11 / 18$ & 61 & \\
$\leq 50$ & $3 / 6$ & 50 & \\
Gender & & & 0.403 \\
$\quad$ Male & $4 / 9$ & 44 & \\
$\quad$ Female & $10 / 15$ & 66.6 & \\
$\quad$ Cytogenetic & & & 0.1 \\
$\quad$ Intermediate risk & $10 / 16$ & 63.6 & \\
$\quad$ High risk & $3 / 6$ & 50 & \\
Marrow blast count & & & 0.678 \\
5-9\% & $6 / 9$ & 60 & \\
10-19\% & $8 / 15$ & 57.1 & \\
\hline
\end{tabular}

$\mathrm{CR}$, complete remission.

signed informed consent prior to the enrollment. Other eligibility criteria were an Eastern Cooperative Oncology Group performance status of $0-2$ and normal cardiac function (23). The clinical parameters assessed at presentation included age, sex, hemoglobin $(\mathrm{Hb})$, white blood cell count (WBC), platelet count and percentage of bone marrow (BM) blasts. A cytogenetic study at diagnosis on BM was performed.

Treatment. All the enrolled patients received induction therapy: This consisted of a combination of HHT $\left(2 \mathrm{mg} / \mathrm{m}^{2}\right.$ intramuscularly twice daily, days $1-3)$ with aclarubicin $\left(12 \mathrm{mg} / \mathrm{m}^{2}\right.$, days 1-7) and $\mathrm{AraC}\left(75 \mathrm{mg} / \mathrm{m}^{2}\right.$ injected subcutaneously twice daily, days 1-7). The response evaluation criteria for the
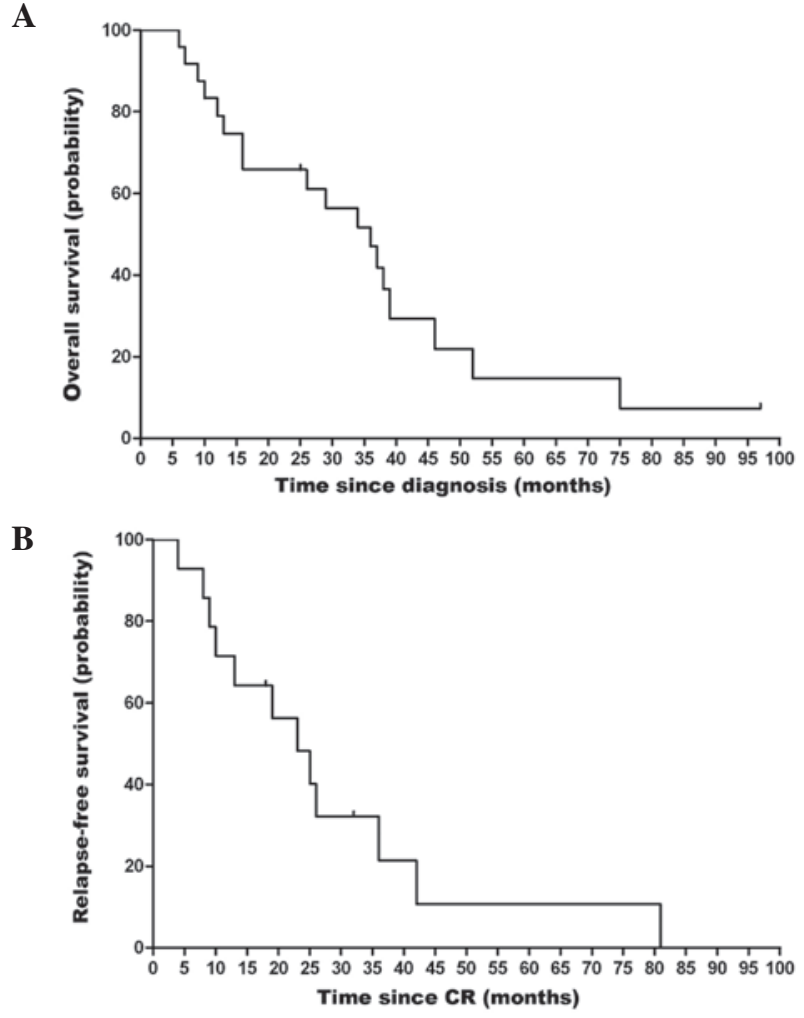

Figure 1. Kaplan Meier curves for survival analyses of the patients. (A) Overall survival of all patients treated with HAA regimen. (B) Relapse-free survival of the patients achieved complete remission.

patients were as follows (24): i) CR was defined as normalization of blood counts with $\geq 1.0 \times 10^{9} / 1, \mathrm{Hb} \geq 110 \mathrm{~g} / \mathrm{dl}$, platelets $\geq 100 \times 10^{9} / 1$, and marrow blasts $\leq 5 \%$ without evidence of dysplasia. ii) Partial remission (PR) was defined as CR except that marrow blasts should reduce by $\geq 50 \%$ compared with the pretreatment levels. iii) Bone marrow $\mathrm{CR}$ was defined as $\leq 5 \%$ marrow blasts without evidence of dysplasia, but complete recovery of blood counts was not achieved. Toxicity of induction therapy was graded according to the National Cancer Institute common toxicity criteria (10). For the patients with CR or PR, a second course was repeated using the same drugs and doses, whereas patients failing to response were offered palliative care. Postremission therapy was offered in rotation after the achievement of CR as following: HAA regimen, $\mathrm{AraC}\left(150 \mathrm{mg} / \mathrm{m}^{2}\right.$, days 1-7) in combination with a second drug, which including daunorubicin (45 mg/m $/ \mathrm{m}^{2}$, days $\left.1-3\right)$, idarubicin $\left(10 \mathrm{mg} / \mathrm{m}^{2}\right.$, days $\left.1-3\right)$, etoposide $\left(75 \mathrm{mg} / \mathrm{m}^{2}\right.$, days $\left.1-5\right)$, mitoxantrone $\left(10 \mathrm{mg} / \mathrm{m}^{2}\right.$, days 1-3), or aclarubicin (12 mg/m², days 1-5). During chemotherapy, the patients received subcutaneous injections of granulocyte colony-stimulating factor at $5 \mathrm{mg} / \mathrm{kg}$, from the day neutrophil count was $<0.5 \times 10^{9} / 1$ until the neutrophil count was $>1.0 \times 10^{9} / 1$ on 3 successive days. Maintenance therapy was administered to all CR patients and continued until the patient was 3 years in remission.

Statistical analysis. Fisher's exact test was used in order to detect the factors that influenced the CR rate. Time-to-event analysis was performed according to the Kaplan-Meier method, and the log-rank test was applied to assess 
A

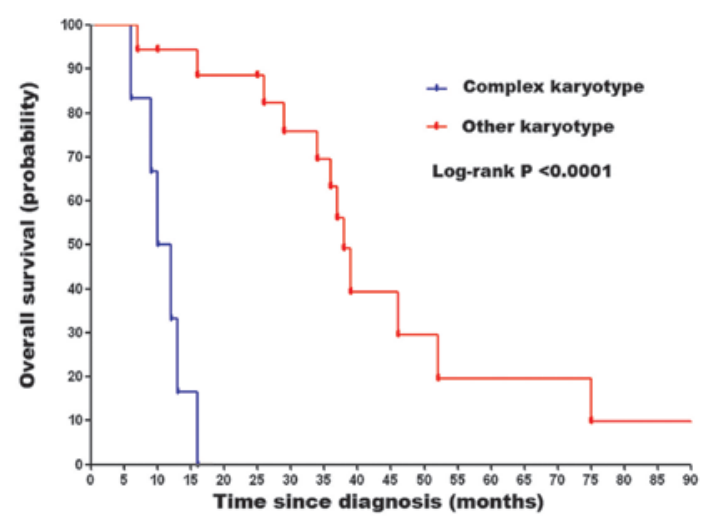

B

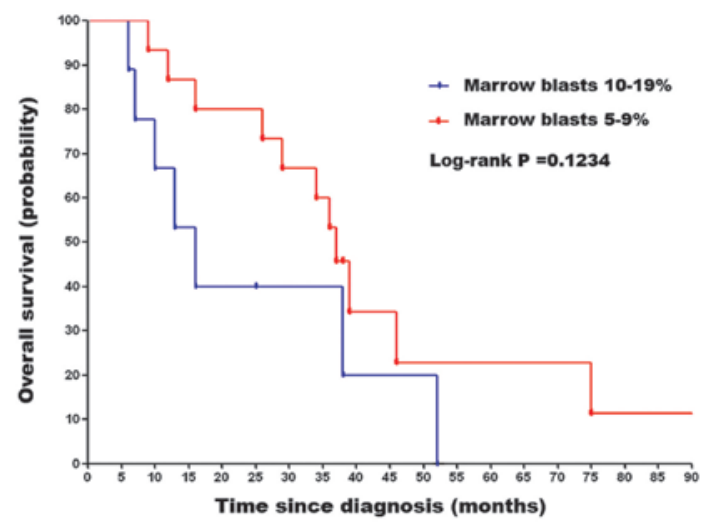

C

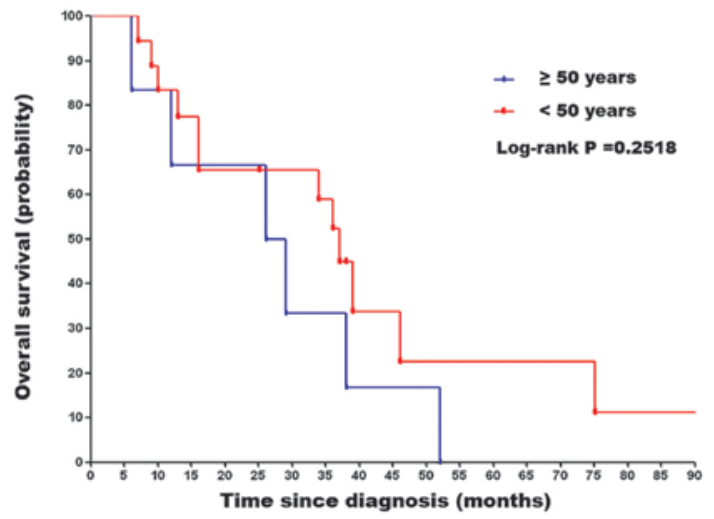

Figure 2. Kaplan Meier curves for overall survival analyses of the patients with different characteristics. (A) Complex abnormal karyotypes vs. other karyotypes [normal, -7, -8, -Y and del(20q)]. (B) High (10-19\%) vs. low $(5-9 \%)$ marrow blasts. (C) High ( $\geq 50$ years) vs. low age ( $<50$ years).

differences between subgroups. All statistical analysis was performed using SPSS 10.0 statistical software (SPSS, Inc., Chicago, IL, USA). $\mathrm{P}<0.05$ was considered to indicate a stastically significant difference.

\section{Results}

Patient characteristics. The baseline characteristics for 24 patients enrolled in the present study are summarized in Table I. The median age of this population was 41 years (range 18-66 years); 6 patients were $>50$ years and 18 patients were $<50$ years. According to the WHO classification, 9 patients had a diagnosis of MDS-RAEB I and 15 had RAEB II. Of the 22 patients who had cytogenetic studies performed, 5 patients $(22.7 \%)$ had intermediate-1, 13 patients
(59.1\%) had intermediate-2, and 4 patients (18.2\%) had high risk MDS, according to the IPSS $(2,13)$.

Response and treatment outcome. A total of 14/24 patients (58.3\%) achieved CR (including bone marrow CR) after the first course of induction treatment, and PR was observed in 5 patients (20.7\%) for an overall response rate of $79 \%$. Univariate analysis of factors influencing CR demonstrated that the age, gender, marrow blast count, and cytogenetic abnormalities of patient had no significant influence on CR rate (Table II).

Fig. 1A shows the overall survival (OS) curve for the 24 patients. The median OS time was 36.2 months [95\% confidence interval (CI), 24.6-47.4 months], and the estimated 3 year OS rate was $45.8 \%$. Univariate analysis of the factors that influenced OS showed a negative impact of complex karyotype $(\mathrm{P}<0.0001$; Fig. 2A). Whereas, marrow blast count and age had no significant impacts on survival (Fig. 2B and C). Fig. 1B shows the relapse-free survival (RFS) curve for the 14 patients who achieved CR. The median RFS for these patients was 27.7 months (95\% CI, 12.9-33.1 months).

Toxicity. Mortality was not associated with treatment with the HAA regimen as an induction therapy. The most common toxicities for cycle 1 were myelosuppression, which occurred in all patients. The nadirs of the neutrophil count and platelet were $0.2 \times 10^{9} / 1$ (range $0.1-0.35 \times 10^{9} / 1$ ) and $4 \times 10^{9} / 1$ (range $\left.2-13 \times 10^{9} / 1\right)$, respectively. The median times to recovery of neutrophil $\left(\geq 1.0 \times 10^{9} / 1\right)$ and recovery of the platelets $\left(\geq 20 \times 10^{9} / 1\right)$ were 24.5 days (95\% CI, 19.9-29.2 days) and 27.2 days (95\% CI, 18.5-35.9 days), respectively. The most common nonhematological toxicities were nausea, emesis, diarrhea and constipation, which were mild with grades I-II.

\section{Discussion}

Higher-risk MDS represents a significant therapeutic treatment challenge for any current chemotherapeutics because of poor responses to combination chemotherapy and shorter survival (25). Previous large studies using various induction regimens containing $\mathrm{AraC}$ in various combinations with idarubicin, fludarabine, topotecan, and cyclophosphamide demonstrated CR rates of $\sim 40-60 \%$ for higher-risk MDS, median OS rate 10.4-13 months, and treatment-related early death $10-20 \%(2,3,26,27)$. In the present study, an HAA regimen was implemented for MDS-RAEB in adults who were aged 18-66 years. A total of 17/22 patients (77.3\%) were scored as higher-risk by IPSS (28). The HAA regimen produced a CR rate of $58.3 \%$, and a median OS of 36.2 months. The median RFS time of the patients who achieved CR was 27.7 months. This compares favorably with the outcomes of traditional intensive chemotherapies reported previously $(26,27,29)$. The patient selection appears to be likely reason for this, since a high proportion of younger patients was included. Previously, Fenaux et al (30) reported that RAEB and predominantly RAEB in transformation (RAEBt) patients treated with an anthracycline-AraC regimen whose CR exceeded 2 years were young with normal karyotypes. Consistent with this result, in the present study, patients with other karyotypes [normal, -7 , $-8,-Y$ and del(20q)], had an improved OS compared with those with complex karyotypes. However, it is worth noting that no 
statistical difference in OS was observed in comparison of high age ( $\geq 50$ years) vs. low age ( $<50$ years), in the present study.

For patients with higher-risk MDS, HAA regimen was found to be effective in inducing durable remissions although the number of patients in the current study was too small to make any definitive conclusions. HHT has also been administered to adults with MDS (7,12,31-33). Daver et al (31) reported that the CR rate was $11 \%$ in patients with intermediate and high risk MDS, who received HHT alone. In addition, combination therapy of HHT and low-dose $\mathrm{AraC}$ has been demonstrated to be an effective strategy for advanced MDS and RAEBt and CR was observed in $46.9 \%$ (32); however, the median OS was shorter (18.2 months). Encouraging results from an open-label, randomized, controlled phase III study using an HAA regimen as an induction therapy in de novo AML patients aged 14-59 years have been reported (34). In that study, 150/206 patients $(73 \%)$ received HAA regimen achieved CR versus 125/205 (61\%) in the patients who were treated with daunorubicin and AraC (DA regimen); 3-year event-free survival was $35.4 \%$ versus $23.1 \%(\mathrm{P}=0.0023)(34)$. These differences appeared to be mainly a consequence of improved outcome in the patients with favorable and intermediate cytogenetics. Moreover, our recent study (35) showed in vitro synergy between HHT and aclarubicin. This combination therapy could synergistically induce the apoptosis of $\mathrm{CD} 34^{+} / \mathrm{CD} 38^{-}$primary AML cells. The probable mechanism of synergy arises from the inhibition of PI3K/Akt and WNT/ $\beta$-catnin signaling pathways, which may be the reason for the clinical benefit of HAA regimen in treating AML and higher-risk MDS.

Despite the presence of myelosuppresion in all of the patients in the present study, which resulted in accompanying risks of infectious complications, the induction mortality was notably low ( 0 by day 30) compared with previous reports in patients with AML who received an HAA regimen (4\% to $5.8 \%$ by day 30) (11,34). One of the major issues concerned with HHT is cardiovascular complications. A previous study showed that a high dose HHT (5-6 mg/m²/day) was associated with severe hypotension and cardiovascular collapse (36). The studies using HAA regimen in AML demonstrated that only $2 \%$ of patients had toxic cardiac effects $(11,34)$. Collectively, these results support the safety of HAA regimen.

Altogether, the data from the present study indicates that the HAA regimen may improve the outcome of de novo higher-risk MDS patients, particularly of those with favorable and intermediate cytogenetics. However, this conclusion was obtained from the comparison with historical controls. Thus, a prospective controlled study is needed to confirm these results.

\section{References}

1. Nimer SD. Myelodysplastic syndromes. Blood 111: 4841-4851, 2008.

2. Fukumoto JS and Greenberg PL. Management of patients with higher risk myelodysplastic syndromes. Crit Rev Oncol Hematol 56: 179-192, 2005.

3. de Witte T, Suciu S, Peetermans M, et al: Intensive chemotherapy for poor prognosis myelodysplasia (MDS) and secondary acute myeloid leukemia (sAML) following MDS of more than 6 months duration. A pilot study by the Leukemia Cooperative Group of the European Organisation for Research and Treatment in Cancer (EORTC-LCG). Leukemia 9: 1805-1811, 1995.

4. Garcia-Manero G and Fenaux P. Hypomethylating agents and other novel strategies in myelodysplastic syndromes. J Clin Oncol 29: 516-523, 2011.
5. Estey EH, Kantarjian HM, O'Brien S, et al: High remission rate, short remission duration in patients with refractory anemia with excess blasts (RAEB) in transformation (RAEB-t) given acute myelogenous leukemia (AML)-type chemotherapy in combination with granulocyte-CSF (G-CSF). Cytokines Mol Ther 1: 21-28, 1995.

6. Estey E, Thall P, Beran M, Kantarjian H, Pierce S and Keating M. Effect of diagnosis (refractory anemia with excess blasts, refractory anemia with excess blasts in transformation, or acute myeloid leukemia [AML]) on outcome of AML-type chemotherapy. Blood 90: 2969-2977, 1997.

7. Feldman EJ, Seiter KP, Ahmed T, Baskind P and Arlin ZA. Homoharringtonine in patients with myelodysplastic syndrome (MDS) and MDS evolving to acute myeloid leukemia. Leukemia 10: 40-42, 1996.

8. Stone RM, Donohue KA, Stock W, et al: A phase II study of continuous infusion homoharringtonine and cytarabine in newly diagnosed patients with chronic myeloid leukemia: CALGB study 19804. Cancer Chemother Pharmacol 63: 859-864, 2009.

9. Quintás-Cardama A, Kantarjian H, Garcia-Manero G, et al: Phase I/II study of subcutaneous homoharringtonine in patients with chronic myeloid leukemia who have failed prior therapy. Cancer 109: 248-255, 2007.

10. Wang J, Lü S, Yang J, et al: A homoharringtonine-based induction regimen for the treatment of elderly patients with acute myeloid leukemia: a single center experience from China. J Hematol Oncol 2: 32, 2009.

11. Jin J, Jiang DZ, Mai WY, et al: Homoharringtonine in combination with cytarabine and aclarubicin resulted in high complete remission rate after the first induction therapy in patients with de novo acute myeloid leukemia. Leukemia 20: 1361-1367, 2006.

12. Xu WL, Jin J and Qian WB. Homoharringtonine in combination with cytarabine and aclarubicin as induction therapy improves remission and survival of patients with higher risk myelodysplastic syndromes. Chin Med J (Engl) 123: 108-110, 2010.

13. Yin S, Wang R, Zhou F, Zhang H and Jing Y. Bcl-xL is a dominant antiapoptotic protein that inhibits homoharringtonine-induced apoptosis in leukemia cells. Mol Pharmacol 79: 1072-1083, 2011.

14. Tujebajeva RM, Graifer DM, Karpova GG and Ajtkhozhina NA. Alkaloid homoharringtonine inhibits polypeptide chain elongation on human ribosomes on the step of peptide bond formation. FEBS Lett 257: 254-256, 1989.

15. Chen R, Guo L, Chen Y, et al: Homoharringtonine reduced Mcl-1 expression and induced apoptosis in chronic lymphocytic leukemia. Blood 117: 156-164, 2011.

16. Jie H, Donghua H, Xingkui X, et al: Homoharringtonine-induced apoptosis of MDS cell line MUTZ-1 cells is mediated by the endoplasmic reticulum stress pathway. Leuk Lymphoma 48: 964-977, 2007.

17. Lindqvist LM, Vikström I, Chambers JM, et al: Translation inhibitors induce cell death by multiple mechanisms and Mcl-1 reduction is only a minor contributor. Cell Death Dis 3: e409, 2012.

18. Meng HT, Li L, Liu H, Wang Y, Li GC and Qian WB. Homoharringtonine acts synergistically with SG235-TRAIL, a conditionally replicating adenovirus, in human leukemia cell lines. Acta Pharmacol Sin 30: 1529-1536, 2009.

19. Meng H, Yang C, Jin J, Zhou Y and Qian W. Homoharringtonine inhibits the AKT pathway and induces in vitro and in vivo cytotoxicity in human multiple myeloma cells. Leuk Lymphoma 49:1954-1962, 2008.

20. Kuroda J, Kamitsuji Y, Kimura S, et al: Anti-myeloma effect of homoharringtonine with concomitant targeting of the myeloma-promoting molecules, Mcl-1, XIAP, and beta-catenin. Int J Hematol 87: 507-515, 2008.

21. Vardiman JW, Harris NL and Brunning RD: The World Health Organization (WHO) classification of the myeloid neoplasms. Blood 100: 2292-2302, 2002.

22. Bennett JM, Catovsky D, Daniel MT, Flandrin G, Galton DA, Gralnick HR and Sultan C: Proposals for the classification of the acute leukaemias. French-American-British (FAB) co-operative group. Br J Haematol 33: 451-458, 1976.

23. Clark TG, Stewart ME, Altman DG, Gabra H and Smyth JF: A prognostic model for ovarian cancer. Br J Cancer. 85: 944-952, 2001.

24. Cheson BD, Greenberg PL, Bennett JM, et al: Clinical application and proposal for modification of the International Working Group (IWG) response criteria in myelodysplasia. Blood 108: 419-425, 2006.

25. Zeidan AM, Linhares Y and Gore SD: Current therapy of myelodysplastic syndromes. Blood Rev 27: 243-259, 2013. 
26. Wattel E, De Botton S, Luc Laï J, et al: Long-term follow-up of de novo myelodysplastic syndromes treated with intensive chemotherapy: incidence of long-term survivors and outcome of partial responders. Br J Haematol 98: 983-991, 1997.

27. Oosterveld M, Muus P, Suciu S, et al; EORTC, EBMT, SAKK, GIMEMA Leukemia Groups and the MD Anderson Cancer Center: Chemotherapy only compared to chemotherapy followed by transplantation in high risk myelodysplastic syndrome and secondary acute myeloid leukemia; two parallel studies adjusted for various prognostic factors. Leukemia 16: 1615-1621, 2002.

28. Greenberg P, Cox C, LeBeau MM, et al: International scoring system for evaluating prognosis in myelodysplasticsyndromes. Blood 89: 2079-2088, 1997.

29. Beran M, Shen Y, Kantarjian H, et al: High-dose chemotherapy in high-risk myelodysplastic syndrome: covariate-adjusted comparison of five regimens. Cancer 92: 1999-2015, 2001.

30. Fenaux P, Morel P, Rose C, Lai JL, Jouet JP and Bauters F: Prognostic factors in adult de novo myelodysplastic syndromes treated by intensive chemotherapy. Br J Haematol 77: 497-501, 1991

31. Daver N, Vega-Ruiz A, Kantarjian HM, et al: A phase II open-label study of the intravenous administration of homoharringtonine in the treatment of myelodysplastic syndrome. Eur J Cancer Care (Engl) 22: 605-611, 2013.
32. Wu L, Li X, Su J, He Q, Zhang X, Chang C and Pu Q: Efficacy and safety of CHG regimen (low-dose cytarabine, homoharringtonine with G-CSF priming) as induction chemotherapy for elderly patients with high-risk MDS or AML transformed from MDS. Cancer Res Clin Oncol 137: 1563-1569, 2011.

33. Wu L, Li X, Su J, et al: Effect of low-dose cytarabine, homoharringtonine and granulocyte colony-stimulating factor priming regimen on patients with advanced myelodysplastic syndrome or acute myeloid leukemia transformed from myelodysplastic syndrome. Leuk Lymphoma 50: 1461-1467, 2009.

34. Jin J, Wang JX, Chen FF, et al: Homoharringtonine-based induction regimens for patients with de-novo acute myeloid leukaemia: a multicentre, open-label, randomised, controlled phase 3 trial. Lancet Oncol 14: 599-608, 2008.

35. Wang L, You LS, Ni WM, et al: $\beta$-Catenin and AKT are promising targets for combination therapy in acute myeloid leukemia. Leuk Res 37: 1329-1340, 2013.

36. Legha SS, Keating M, Picket S, Ajani JA, Ewer M and Bodey GP. Phase I clinical investigation of homoharringtonine. Cancer Treat Rep 68: 1085-1091, 1984. 